\title{
High-throughput screening and whole genome sequencing identifies an antimicrobially active inhibitor of Vibrio cholerae
}

\author{
Galina Sergeev', Sambit Roy ${ }^{1}$, Michael Jarek ${ }^{1}$, Viktor Zapolskii ${ }^{3}$, Dieter E Kaufmann ${ }^{3}$, Ranjan K Nandy ${ }^{2 *}$ \\ and Werner Tegge ${ }^{1 *}$
}

\begin{abstract}
Background: Pathogenic serotypes of Vibrio cholerae cause the life-threatening diarrheal disease cholera. The increasing development of bacterial resistances against the known antibiotics necessitates the search for new antimicrobial compounds and targets for this pathogen.

Results: A high-throughput screening assay with a Vibrio cholerae reporter strain constitutively expressing green fluorescent protein (GFP) was developed and applied in the investigation of the growth inhibitory effect of approximately 28,300 structurally diverse natural compounds and synthetic small molecules. Several compounds with activities in the low micromolar concentration range were identified. The most active structure, designated vz0825, displayed a minimal inhibitory concentration (MIC) of $1.6 \mu \mathrm{M}$ and a minimal bactericidal concentration (MBC) of $3.2 \mu \mathrm{M}$ against several strains of $V$. cholerae and was specific for this pathogen. Mutants with reduced sensitivity against vz0825 were generated and whole genome sequencing of 15 pooled mutants was carried out. Comparison with the genome of the wild type strain identified the gene VC_A0531 (GenBank: AE003853.1) as the major site of single nucleotide polymorphisms in the resistant mutants. VC_A0531 is located on the small chromosome of $V$. cholerae and encodes the osmosensitive $\mathrm{K}^{+}$-channel sensor histidine kinase $(\mathrm{KdpD})$. Nucleotide exchange of the major mutation site in the wild type strain confirmed the sensitive phenotype.

Conclusion: The reporter strain MO10 pG13 was successfully used for the identification of new antibacterial compounds against $V$. cholerae. Generation of resistant mutants and whole genome sequencing was carried out to identify the histidine kinase KdpD as a novel antimicrobial target.
\end{abstract}

Keywords: Vibrio cholerae, Small molecules, Histidine kinase inhibitor, KdpD, whole genome sequencing

\section{Background}

Vibrio cholerae, a Gram-negative rod-shaped bacterium belonging to the family Vibrionaceae, induces the acute diarrheal disease cholera. Cholera has pandemic properties and appears mainly in third world countries with estimated 3-5 million cases and more than 100,000 deaths per year [1]. The major pathogenic strains belong to the serogroups $\mathrm{O} 1$ and O139. Infections are treated by oral or intravenous rehydration therapy, which is complemented

\footnotetext{
* Correspondence: nandy_rk@hotmail.com; werner.tegge@helmholtz-hzi.de ${ }^{2}$ National Institute of Cholera and Enteric Diseases (NICED), P-33, CIT Road, Scheme XM Beliaghata, Kolkata 700 010, India

${ }^{1}$ Department of Chemical Biology, Helmholtz Centre for Infection Research (HZl), Inhoffenstraße 7, D-38124 Braunschweig, Germany

Full list of author information is available at the end of the article
}

in severe cases with antibiotics to shorten the duration of the clinical symptoms and to reduce the spreading. Longterm and extensive use of antibiotics has led to resistance development. A growing problem is the emergence of multidrug resistant pathogenic $V$. cholerae strains against which therapeutic options are more and more limited [2]. Due to this development the availability of novel therapeutic options is urgently needed.

In the present study we have developed a highthroughput screening (HTS) assay that utilizes a V. cholerae reporter strain constitutively expressing green fluorescence protein and screened approximately 28,300 compounds from six different chemical structural groups in a growth inhibition assay. Several active molecules were identified

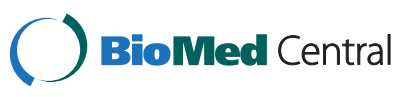


which are active in suppressing growth of $V$. cholerae in vitro. $V$. cholerae mutants resistant to the most potent molecule were generated. Whole-genome sequencing and comparative analysis of the mutant to the wild type strain was carried out. The apparent target of the most active compound was identified to be the osmosensitive $\mathrm{K}^{+}$-channel sensor histidine kinase KdpD that apparently exerts certain essential function in this pathogen.

\section{Results}

\section{HTS assay for inhibitors of $V$. cholerae viability}

Green fluorescence producing plasmid pG13 was electroporated into $V$. cholerae strain MO10 and the transformants were selected on LB agar plates containing kanamycin $(\mathrm{Km}, 30 \mu \mathrm{g} / \mathrm{ml})$. Transfer of the plasmid pG13 conferred green fluorescence phenotype in $V$. cholerae $\mathrm{O} 139$ strain MO10. The screening assay was optimized in 96- and 384-well microtiter plates (MTP). To differentiate between active and non-active compounds and as controls for the functionality of the assay, ciprofloxacin (Cip, $100 \mu \mathrm{M}$ ) and dimethyl sulfoxide (DMSO, $1 \%)$ were included on each plate. DMSO had no growth reducing effect at concentrations up to $1 \%$. The evaluation of the effect of compounds on the growth of strain MO10 pG13 was carried out after $24 \mathrm{~h}$ of incubation, with measurement of absorbance at $600 \mathrm{~nm}$ in combination with fluorescence determination (Figure 1). In the screening campaigns of the six different substance collections with 28,300 compounds in total, Z'-values between 0.5 and 0.9 with a mean of 0.8 were obtained, which is an indication of a reliable performance of the assay [3].

The six groups of screening compounds consisted of: i) the commercially available LOPAC library (a collection of pharmaceutically active compounds); ii) and iii) the EMC (Echaz Microcollection) and CDI collections (Chemical Diversity Lab), which contain small organic molecules that were mainly generated by combinatorial synthesis; iv) the VAR collection (various sources), which is unique at the HZI and consists of small organic molecules that were synthesized by cooperating chemists; v) the $\mathrm{NCH}$ collection (natural compounds), which is also unique at the HZI and consists of purified secondary metabolites from myxobacteria. It included potent agents with already known antimicrobial or antiproliferative activity, e.g. epothilon, which has been developed into a therapeutic agent against breast cancer [4,5]; and finally vi) collections of linear and cyclic peptides with a length of seven or eight D- or Lamino acids were investigated [6]. The compounds were used in one defined concentration between 20 to $50 \mu \mathrm{M}$ in the initial screening. An overview of the growth-reducing activities of the six different substance collections is shown in Figure 2 and in Table 1. The threshold for active compounds was defined at a minimum growth reduction of
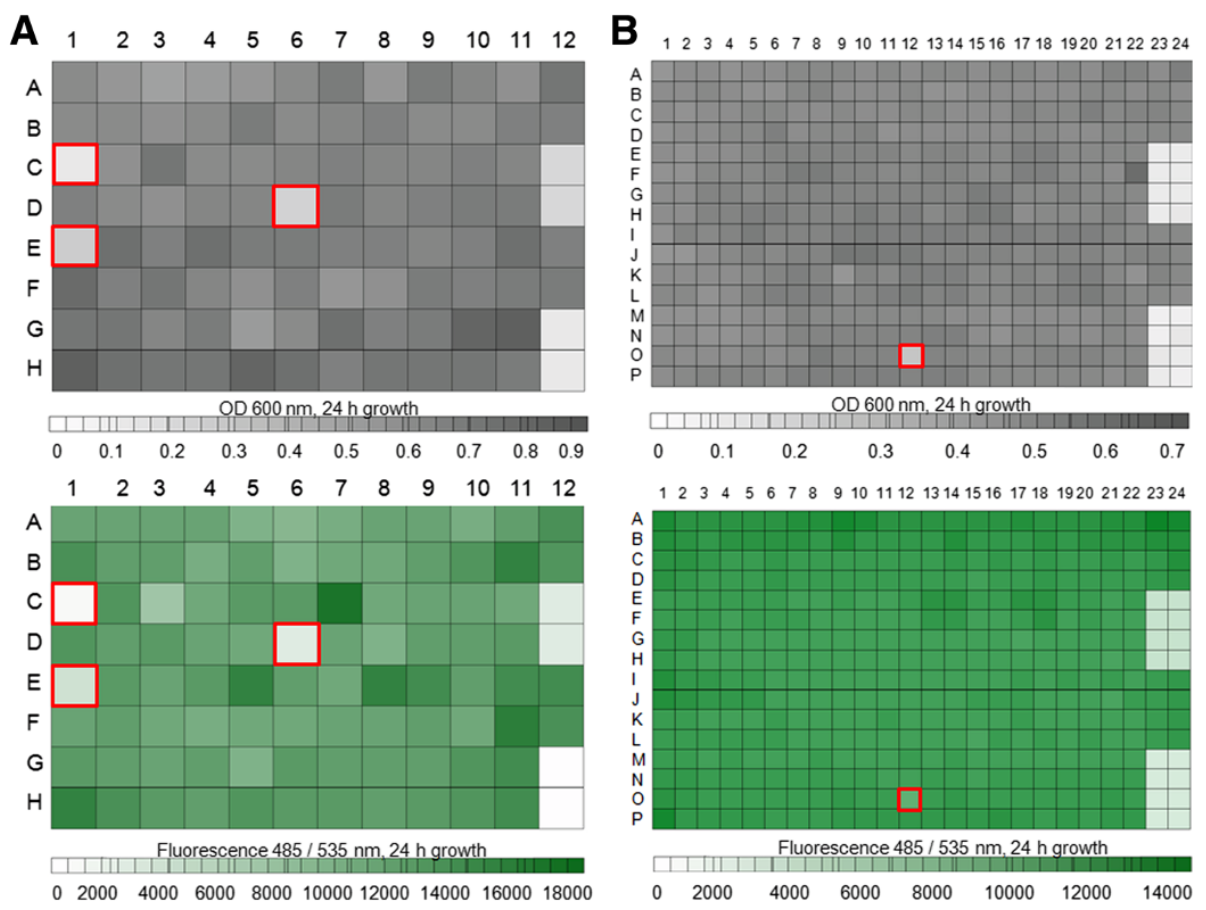

Figure 1 HTS assay. Growth of V. cholerae MO10 pG13 strain in 96- (A) and 384-well MTP (B) in the presence of test compounds and controls. (A): 12 A-B: 1\% DMSO, 12C-D: $100 \mu \mathrm{M}$ ciprofloxacin, 12 E-F: no addition of compounds, 12 G-H: sterile medium. (B): 23 A-D and 24 A-D: $1 \%$ DMSO, $23 \mathrm{E}-\mathrm{H}$ and $24 \mathrm{E}-\mathrm{H}: 100 \mathrm{\mu M}$ ciprofloxacin, $23 \mathrm{~J}-\mathrm{M}$ and $24 \mathrm{~J}-\mathrm{M}$ : no addition of compounds, $23 \mathrm{M}-\mathrm{P}$ and $24 \mathrm{M}-\mathrm{P}$ : sterile medium. Upper panels: absorbance at $600 \mathrm{~nm}$; lower panels: fluorescence $(485 / 535 \mathrm{~nm})$. Wells framed in red indicate active compounds. 


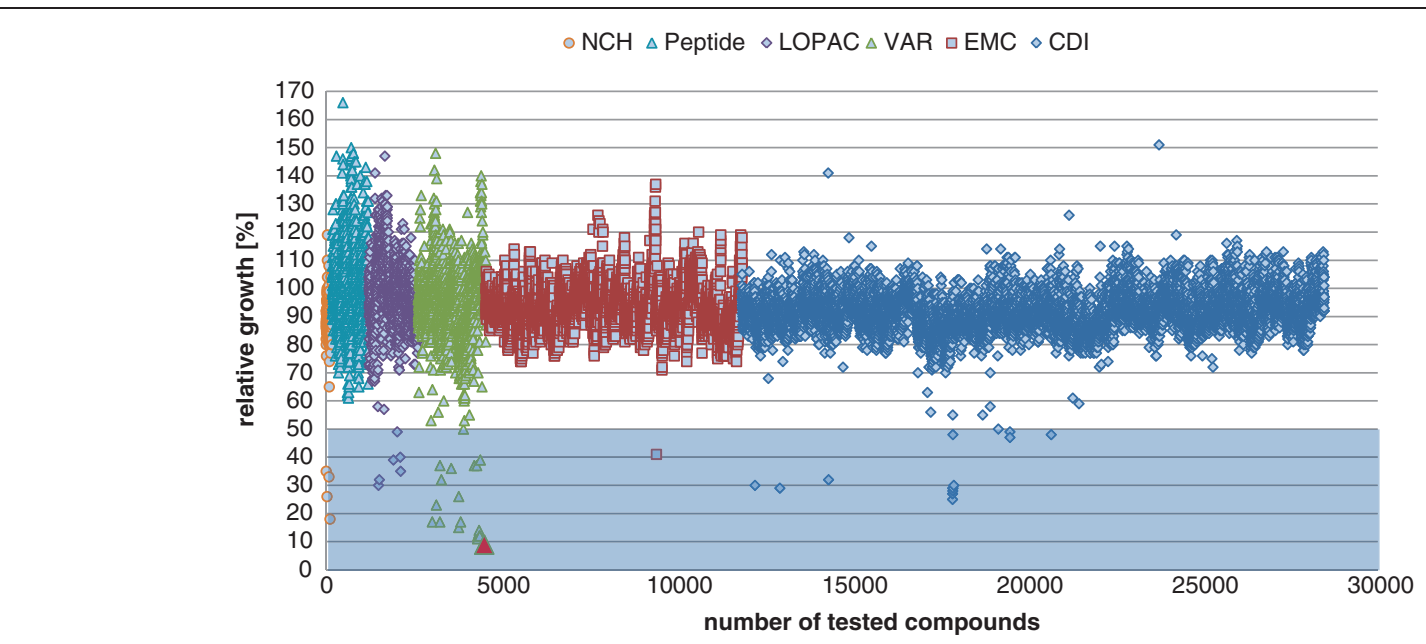

Figure 2 Screening results. Summary of the initial screening results for novel antibacterial compounds. The tested compounds came from the NCH, Peptide, LOPAC, VAR, EMC and CDI collections. The shaded area highlights the activities that were defined as initial hits. The most active compound, vz0825, stemming from the VAR collection, is highlighted in red.

$50 \%$ in comparison to the DMSO control, which resulted in a suitable initial hit rate. The smallest of the six collections, the NCH collection of 154 compounds, showed the most active molecules with 32.5 hits per 1,000 substances. Several of these molecules displayed antibacterial activities that have been known before [7]. The VAR library consists of molecules with predominantly unexplored activities and contained 8.8 antibacterial compounds per 1,000 molecules. With 17 hits this collection contained the highest number of antibacterial molecules in total.

In total 42 hits were identified in the initial screening campaign. These initial hits were reevaluated in different concentrations by using $V$. cholerae strains and several other Gram-positive and Gram-negative pathogenic bacteria. After these reevaluations, the number of active compounds was reduced to three most promising agents with the designations vz0825, vz0500 and 1541-0004. The former two compounds are derived from the VAR library, the last one from the commercially available CDI library. The chemical structures are shown in Figure 3.
MIC and MBC values of the most active substances

The two pathogenic $V$. cholerae O1 type stains N16961 and NM06-058 were used to determine the MIC and $\mathrm{MBC}$ values for the compounds vz0825, vz0500 and 1541-0004 (Table 2). V. cholerae N16961 belongs to biotype El Tor which caused the seventh pandemic [8] and was isolated in 1971. V. cholerae NM06-058 was isolated in 2006 in Kolkata from a cholera patient and represents the altered El Tor biotype. The active compounds inhibited growth of both strains equipotent at low micromolar concentrations with MIC values of $1.6 \mu \mathrm{M}, 3.1 \mu \mathrm{M}$ and $6.3 \mu \mathrm{M}$, respectively. In order to obtain reliable data, bactericidal activities were determined after 2, 6 and 24 hours. All three compounds killed the bacteria at low micromolar concentrations, only slightly above the respective MIC values (Table 2). Further nine $V$. cholerae strains belonging to the O1, O139 and non O1/O139 serogroups (Table 3) (three strains of each serogroup) were testes with compound vz0825, which is active against all tested strains with MIC values between 0.4 and $3.1 \mu \mathrm{M}$. Overall vz0825 was the most active substance.

Table 1 Summary of the screening for growth-reducing compounds

\begin{tabular}{|c|c|c|c|c|c|c|c|}
\hline \multirow{2}{*}{$\begin{array}{l}\text { Substance collection } \\
\text { (number of compounds) }\end{array}$} & \multicolumn{5}{|c|}{ Number of active compounds at different growth reduction rates } & \multirow[t]{2}{*}{$\Sigma$} & \multirow{2}{*}{$\begin{array}{c}\text { Hit } \\
\text { rate/1,00c }\end{array}$} \\
\hline & $50-60 \%$ & $60-70 \%$ & $70-80 \%$ & $80-90 \%$ & $90-100 \%$ & & \\
\hline $\mathrm{NCH}(154)$ & 0 & 2 & 1 & 2 & 0 & 5 & 32.5 \\
\hline Peptide $(1,045)$ & 0 & 0 & 0 & 0 & 0 & 0 & 0 \\
\hline LOPAC $(1,408)$ & 2 & 4 & 0 & 0 & 0 & 6 & 4.3 \\
\hline $\operatorname{VAR}(1,936)$ & 1 & 5 & 2 & 8 & 1 & 17 & 8.8 \\
\hline $\operatorname{EMC}(7,304)$ & 1 & 0 & 0 & 0 & 0 & 1 & 0.1 \\
\hline CDI $(16,608)$ & 5 & 3 & 5 & 0 & 0 & 13 & 0.8 \\
\hline 28,324 & & & & & & 42 & 1.6 \\
\hline
\end{tabular}




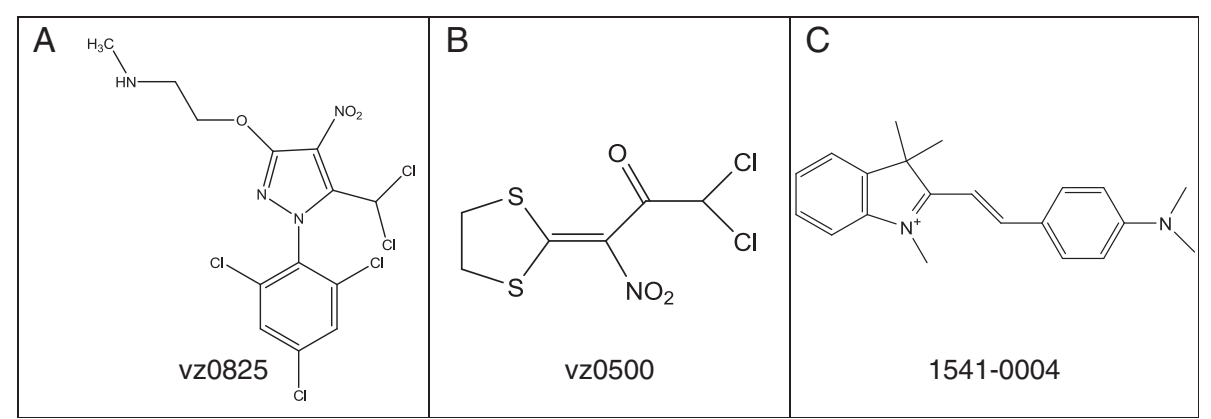

Figure 3 Chemical structures. Most active compounds of V. cholerae growth inhibition. Panel A: compound vz0825; Panel B: compound vz0500; Panel C: compound 1541-0004.

The antibacterial specificity of the active substances was investigated with different Gram-positive and Gram-negative pathogenic bacteria, which are able to induce serious gastrointestinal infections in humans (Table 4). Apparently, the antimicrobial activity of the three substances was limited to $V$. cholerae, only compound 1541-0004 also displayed a moderate activity against $S$. aureus with an MIC of $6.3 \mu \mathrm{M}$.

\section{Cytotoxicity determination via MTT-assay}

In vitro cytotoxicity determination by MTT test with mammalian cells is one of the standard procedures for the evaluation of new active agents [10]. The well established assay was carried out with the permanent mouse fibroblast cell line L929 according to a published procedure [11] with some modifications [12]. In the assay cell viability is determined by the reduction of the yellow MTT (3-(4,5-dimethylthiazol-2-yl)-2,5-diphenyltetrazoliumbromid) to the violet formazan by the action of ER- and mitochondrial enzymes. Concentrations of the active compounds vz0825, vz0500 and 1541-0004 from 0.003 to $370 \mu \mathrm{M}$ were used and effects on the fibroblasts were analyzed after 24 hours and 5 days of incubation. The $\mathrm{IC}_{50}$ values are shown in Table 5 . The

Table 2 MIC and MBC values for the most active compounds against $V$. cholerae

\begin{tabular}{|c|c|c|c|c|c|}
\hline \multirow[b]{2}{*}{ V. cholerae strain } & & \multirow[b]{2}{*}{$\begin{array}{l}\text { Incubation } \\
\text { time }\end{array}$} & \multicolumn{3}{|c|}{ Concentration $[\mu \mathrm{M}]$} \\
\hline & & & vz0825 & vz0500 & $1541-0004$ \\
\hline \multirow[t]{4}{*}{ N16961 } & MIC & $24 \mathrm{~h}$ & 1.6 & 3.1 & 6.3 \\
\hline & $\mathrm{MBC}$ & $2 \mathrm{~h}$ & 50 & 50 & 50 \\
\hline & & $6 \mathrm{~h}$ & 12.5 & 6.3 & 6.3 \\
\hline & & $24 \mathrm{~h}$ & 6.3 & 6.3 & 6.3 \\
\hline \multirow[t]{4}{*}{ NM06-058 } & MIC & $24 \mathrm{~h}$ & 1.6 & 3.1 & 6.3 \\
\hline & MBC & $2 \mathrm{~h}$ & 50 & 50 & 6.3 \\
\hline & & $6 \mathrm{~h}$ & 12.5 & 6.3 & 6.3 \\
\hline & & $24 \mathrm{~h}$ & 1.6 & 6.3 & 6.3 \\
\hline
\end{tabular}

two most active compounds vz0825 and vz0500 showed cytotoxic (inhibition after 24 hours of incubation) and anti-proliferative (inhibition after 5 days of incubation) $\mathrm{IC}_{50}$ values at low micromolar concentrations. Compound 1541-0004 is less cytotoxic, but has also a strong antiproliferative activity.

\section{Generation of resistant mutants against vz0825}

Mutants against vz0825 were generated by selection of variants of the wild type strain NM06-058 that are able to grow on agar plates containing $8 \mu \mathrm{M}$ vz0825. After one round of selection, 15 resistant mutants were picked and analyzed individually. They displayed 4-16 fold reduced sensitivities (MIC $6.3-25 \mu \mathrm{M}$ ) against vz0825 compared to the wild type strain. In order to obtain an indication if vz0825 has a mode of action that is different from standard antimicrobials, eight established antibiotics against the major different antibacterial targets were tested with the resistant mutants. The addressed targets and their inhibitors were i) cell wall synthesis (ampicillin), ii) protein biosynthesis (tetracycline), iii) DNA-replication (ciprofloxacin), iv) DNA-dependent RNA polymerase (rifampicin), v) translation (chloramphenicol, erythromycin) and vi) synthesis of folic-acid (trimethoprim/sulfamethoxazol).

The $V$. cholerae wild type strain NM06-058 and resistant mutants did not show differences in their MIC values against all tested antibiotics (data not shown), suggesting that vz0825 has a mode of action that is different from the classical antibiotics.

\section{Target identification}

This result initiated a further investigation of the mode of action of vz0825 by the comparative genome sequence analysis approach. The method makes use of whole genome sequence analysis of resistant mutants that were generated against an active compound and the comparison of the genome of the wild type and the mutant strain [13]. The genomes of the 15 resistant $V$. cholerae mutants were isolated, pooled and analyzed via paired-end sequencing. In parallel, the genome of the wild type strain from which 
Table 3 Strains, cells, plasmids and primers used for this study

\begin{tabular}{|c|c|c|}
\hline Strain, cell, plasmid, primer & Relevant description/sequence & Reference or source \\
\hline \multicolumn{3}{|l|}{ Strains } \\
\hline \multicolumn{3}{|l|}{ V. cholerae } \\
\hline MO10 pG13 & O139 containing pG13 & This study \\
\hline N16961 & Wild type, O1, El Tor, Inaba & Makassar (1971), clinical isolate [8] \\
\hline NM06-058 & Wild type, O1, El Tor, Ogawa & Kolkata (1996), clinical isolate \\
\hline NM06-058 T283M & $\begin{array}{l}\text { Contains a point mutation in gene } \\
\text { VC_A0531 on AA position } 283\end{array}$ & This study \\
\hline RKI-ZBS2-A310-3 & Isolate, O1, El Tor, Inaba & RKI \\
\hline RKI-ZBS2-A310-12 & Isolate, O1, El Tor, Ogawa & RKI \\
\hline RKI-ZBS2-A198-1 & Isolate, O1, El Tor, Ogawa & RKI \\
\hline RKI-ZBS2-A310-25 & Isolate, O139, El Tor & RKI \\
\hline RKI-ZBS2-A186-9 & Isolate, O139, El Tor & RKI \\
\hline RKI-ZBS2-186-10 & Isolate, O139, El Tor & RKI \\
\hline RKI-ZBS2-A220-1 & Isolate, Non 01/0139 & RKI \\
\hline RKI-ZBS2-A222-1 & Isolate, Non 01/0139 & RKI \\
\hline RKI-ZBS2-A227-1 & Isolate, Non 01/0139 & RKI \\
\hline \multicolumn{3}{|l|}{ Gram-negative } \\
\hline Acinetobacter baumannii & ATCC 30007 & DSMZ \\
\hline E. coli & ESBL, 5044257621-1 & $\mathrm{HZl}$ \\
\hline E. coli & ETEC & NICED \\
\hline E. coli & S17-1 & $\mathrm{HZl}$ \\
\hline Klebsiella pneumoniae & 50219455 & $\mathrm{HZl}$ \\
\hline Pseudomonas aeruginosa & 90013687 & $\mathrm{HZl}$ \\
\hline Salmonella typhimurium & & NICED \\
\hline Shigella boydii & & NICED \\
\hline Shigella flexneri & & NICED \\
\hline \multicolumn{3}{|l|}{ Gram-positive } \\
\hline Enterococcus faecalis & ATCC 20212 & $\mathrm{HZl}$ \\
\hline Staphylococcus aureus & MRSA, N315 & $\mathrm{HZl}$ \\
\hline \multicolumn{3}{|l|}{ Cell line } \\
\hline L929 & Mouse fibroblastic cell line & Derived from commercial source, DSMZ: ACC 2 \\
\hline \multicolumn{3}{|l|}{ Plasmid } \\
\hline pG13 & $\begin{array}{l}\text { Plasmid containing the constitutive expressing } \\
\text { G13 promoter- and gfp-gene sequence, } \\
\text { ligated in pFPV27 vector, }\left(\mathrm{Km}^{r}\right)\end{array}$ & [9] \\
\hline pEX18Ap & $\begin{array}{l}\text { Plasmid containing Amp }{ }^{r} \text { gene } \beta \text {-lactamase, } \\
\text { the sacB gene encoding the levansucrase }\end{array}$ & $\mathrm{HZl}$ \\
\hline \multicolumn{3}{|l|}{ Oligonucleotide primer } \\
\hline VC_A0531_forw2 & TCACGAACCAACAGGATTAAG & Used for colony PCR and sequencing of the products \\
\hline VC_A0531_rev2 & CGGTTAAAGTGGTAGCAGAG & Same as above \\
\hline Mut_forw_1 & ACATCATCTAGAGCAGCAGCAACACAAGA (Xbal) & Used for generation of the point mutation \\
\hline Mut_rev_1 & ATCGCGCCAAGCGGCATTTTTAGATCG & Same as above \\
\hline Mut_forw_2 & CGATCTAAAAATGCCGCTTGGCGCGAT & Same as above \\
\hline Mut_rev_2 & ACATCAAAGCTTAACATGCGCCACCAGAC (HindIII) & Same as above \\
\hline kdpD_del_forw_1 & ACATCATCTAGAGGAATCCATCAAAGAAA (Xbal) & Used for generation of the deletion mutation of $k d p D$ \\
\hline
\end{tabular}


Table 3 Strains, cells, plasmids and primers used for this study (Continued)

\begin{tabular}{lll}
\hline$k d p D$ del_rev_1 & ACAGGATTAAGAAGCAATGAACAGTGAAATTAAGATCCTC & Same as above \\
$k d p D$ del_forw_2 & GAGGATCTTAATTTCACTGTTCATTGCTTCTTAATCCTGT & Same as above \\
$k d p D$ del_rev_2 & ACATCACTGCAGAACACAAGATCCAACAC (Pstl) & Same as above \\
\hline
\end{tabular}

the resistant mutants have been generated was also sequenced by the same method. The alignment and annotation of both probes was based on the published genome of V. cholerae strain N16961 (chromosome 1: AE003852, chromosome 2: AE003853 in NCBI) [14]. As shown in Table 6, approximately $98 \%$ and $94 \%$ of the fragments from the mutant-pool and the wild type, respectively, could be aligned. The alignment was carried out via the application of CLC Genomics Workbench V. 4.7.2 software. The algorithm to search for crucial distinctions were parameters like single nucleotide polymorphism (SNP) and deletion and insertion polymorphism (DIP), where one nucleotide was affected with a minimal mutation frequency of $30 \%$.

Under those conditions, the comparison of the wild type and the pooled sequences from the mutants showed only one significant mutation, this was located at position 848 in gene VC_A0531 and was present in about 30\% (precisely $29.1 \%$ ) of the sequenced fragments. These mutants have the nucleobase thymine instead of cytosine on position 848. The point mutation of this nucleobase leads to an exchange of threonine to methionine on position 283 (T283M) of the expressed protein.

The gene VC_A0531 (GenBank: AE003853.1) is located on the small chromosome of $V$. cholerae and encodes a sensor histidine kinase, which is the homologous to KdpD of $E$. coli and is responsible for osmotic potassium regulation in the bacterial cell [15]. In addition to the whole

Table 4 MIC values of active compounds for different pathogenic bacteria

\begin{tabular}{|c|c|c|c|}
\hline \multirow[b]{2}{*}{ Bacterial strain } & \multicolumn{3}{|c|}{$\mathrm{MIC}[\mu \mathrm{M}]$} \\
\hline & vz0825 & vz0500 & $1541-0004$ \\
\hline \multicolumn{4}{|l|}{ Gram-negative } \\
\hline Acinetobacter baumannii & 50 & $>>100$ & $>100$ \\
\hline Escherichia coli, ESBL & $>100$ & $>>100$ & $>100$ \\
\hline Escherichia coli, ETEC & $>>50$ & $>>50$ & $>50$ \\
\hline Klebsiella pneumoniae & 100 & $>100$ & 100 \\
\hline Pseudomonas aeruginosa & $>>100$ & $>>100$ & $>>100$ \\
\hline Salmonella typhimurium & $>>50$ & $>>50$ & $>>50$ \\
\hline Shigella boydii & $>>50$ & $>>50$ & $>50$ \\
\hline Shigella flexneri & $>>50$ & $>>50$ & $>50$ \\
\hline \multicolumn{4}{|l|}{ Gram-positive } \\
\hline Enterococcus faecalis & 50 & $>>100$ & $>100$ \\
\hline Staphylococcus aureus, MRSA & 50 & 100 & 6.3 \\
\hline
\end{tabular}

genome pool sequencing, the gene VC_A0531 $(k d p D)$ of the 15 mutants was analyzed individually by PCR amplification. 4 of the 15 mutants, corresponding to $26.7 \%$, had the same mutation on reference position 848 of the gene $k d p D$ that was identified in the whole genome pool sequencing. Another four of the mutants showed point mutations at other positions of the $k d p D$ gene (Table 7).

\section{Sensitivity of strain NM06-058 T283M against vz0825}

A strain containing the point mutation T283M in the $k d p D$ gene was generated by site-directed mutagenesis. Successful cloning was verified by a PCR amplification of the affected gene and the sequencing of the fragment. The mutant was selected on LB-agar plates containing vz0825 at $16 \mu \mathrm{M}$ concentration, which is 10-times higher than the MIC of the wild type strain. A growth analysis with this strain was carried out in vz0825 supplemented LBmedium and in T-medium with different potassium and sodium ion concentrations (Figure 4). Overall, growth of the T283M mutant was much less effected by vz0825 in comparison to the wild type strain. Sensitivity of the T283M mutant against compounds vz0500 and 15410004 did not differ from the wild type strain NM06-058 (data not shown).

\section{Attempts to construct a $k d p D$ knockout mutant}

For a further elucidation of the effect of vz0825, the construction of a $V$. cholerae $k d p D$ knockout mutant was attempted. If $\mathrm{KdpD}$ is a major target of compound vz0825, the $V$. cholerae $k d p D$ knockout mutant should be insensitive to the compound, unless the protein itself and its function are essential for the viability of the bacteria. The cloning procedure delivered the expected plasmid construct according to sequencing. The plasmid was successfully transformed into the $E$. coli strain S17-1, according to the acquirement of ampicillin resistance, which is located on the plasmid pEX18Ap and also according to PCR amplification of the construct. The conjugation of the

Table 5 Cytotoxic (24 h) and antiproliferative (5 d) activity of the most active compounds according to MTT test with L929 cells

\begin{tabular}{lcc}
\hline Compound & \multicolumn{2}{c}{$\mathbf{I C}_{\mathbf{5 0}}[\boldsymbol{\mu M}]$} \\
\cline { 2 - 3 } & $\mathbf{2 4} \mathbf{h}$ & $\mathbf{5 ~ d}$ \\
\hline VZ0825 & 14 & 6 \\
VZ0500 & 3 & 1 \\
$1541-0004$ & 170 & 14 \\
\hline
\end{tabular}


Table 6 Summarized statistics of genome sequencing

\begin{tabular}{lccc}
\hline & $\begin{array}{c}\text { Number of } \\
\text { fragments }\end{array}$ & $\begin{array}{c}\text { Average } \\
\text { length [bp] }\end{array}$ & $\begin{array}{c}\text { Total base } \\
\text { number }\end{array}$ \\
\hline wt genome & $11,260,864$ & 76 & $855,825,664$ \\
Fragments & $10,574,557(93.9 \%)$ & 76 & $803,666,332$ \\
Identified & $686,307(6.1 \%)$ & 76 & $52,159,332$ \\
Non-identified & & & \\
Genome-pool & $35,196,596$ & 72.36 & $2,546,713,435$ \\
Fragments & $34,210,563(97.8 \%)$ & 72.43 & $2,477,950,102$ \\
Identified & $986,033(2.8 \%)$ & 69.74 & $68,763,333$ \\
Non-identified & 2 & $2,016,732$ & $4,033,460$ \\
Reference & 2 &
\end{tabular}

Reference genome came from V. cholerae strain N16961 [14].

transformed E. coli with $V$. cholerae and the following selection on LB agar plates supplemented with carbenicillin (Carb) and $\mathrm{Km}$ did not lead to clones with a deleted VC_A0531 gene, even after several modifications of the protocol. A possible explanation is that the gene product $\mathrm{KdpD}$ is indeed essential for $V$. cholerae, in agreement with KdpD being a prime target of vz0825.

\section{Discussion}

A HTS assay for small molecule inhibitors of $V$. cholerae was developed and validated using a viability phenotype of $V$. cholerae that constitutively expresses green fluorescence. The assay is reliable, reproducible and simple to perform. During the development of the reporter strain, two reference strains of $\mathrm{O} 1$ serogroup belonging to biotypes O395 (classical) and N16961 (El Tor) were included along with the $\mathrm{O} 139$ strain MO10. The green fluorescence producing plasmid pG13 was electroporated into the three strains. During initial standardization experiments it was observed that the strain MO10 pG13 produced much greater level of green fluorescence as compared to other two strains (data not shown). For this reason strain MO10 pG13 was used in the screening experiments.

A data bank search in SciFinder for the most active compounds vz0825 and vz0500 did not reveal predescribed antibacterial activities of the compounds with structural similarities above 70\%. Compound 1541-0004, stemming from the commercial CDI collection, belongs to the group of styryl dyes, which have already in 1966 been shown to possess antimicrobial effects against the plant pathogen Xanthomonas oryza [16]. The $\mathrm{IC}_{50}$ value for acute cytotoxicity of compound vz0825 in an MTT test was approximately 17-times higher than its MIC value. For vz0500, both $\mathrm{IC}_{50}$ and MIC values were about equal. For compound 1541-0004 the $\mathrm{IC}_{50}$ value for cytotoxicity was approximately 27-times higher than the MIC value. Although the identified compounds exhibited antimicrobial activities at low concentrations, the toxicities render them unsuitable for direct clinical application. Thus, the compounds may serve as pharmaceutical leads and modifications via the methods of medicinal chemistry may lead to better properties.

The elucidation of the mode of action of new antimicrobials can be a tedious and time consuming effort and can require the application of a variety of biochemical and molecular methods $[17,18]$. Due to the advances in genome sequencing instrumentation and methodology, an innovative new option has become available recently. It employs genomic sequence comparison of resistant mutants with wild type strains and has been successfully applied for target identification in a limited number of previous investigations by other researchers [13]. As we have used NM06-058 for the evaluation of the active compounds, we have used the same strain to create resistant mutants against vz0825. The $V$. cholerae strain NM06-058 was isolated from hospitalized diarrhea cases during 2006 at Kolkata, India. This strain along with other $V$. cholerae strains isolated during 2006 was studied for the expression of cholera toxin $(\mathrm{CT})$ and it was identified that NM06-058 is capable of producing a higher amount of CT in vitro compared to other strains and to reference $V$. cholerae O1 El Tor strain N16961. Based on the high virulence expression, this strain was selected for our investigations. Clinical $V$. cholerae $\mathrm{O} 1$ strains isolated at Kolkata during and after 1995 belonged to altered El Tor biotypes [19]. Thus it can be considered that strain NM06-058 represents the altered $V$. cholerae El Tor biotype, which is still the prevailing type among cholera cases.

The generation of mutants that were resistant against vz0825 was straightforward in this study by plating the wild type strain on agar plates containing the active compound at 5-times the MIC value of the wild type. The successful generation of resistant mutants with only one

Table 7 Modifications detected in gene VC_A0531 (kdpD) by PCR analysis of 15 resistant mutants (AA, amino acid)

\begin{tabular}{cccccccccc}
\hline & Nucleotide pos. & Ref. allel & Mut. allel & Number of mutants & Codon old & Codon new & AA pos. & AA old & AA new \\
\hline 1 & 218 & T & C & 1 & CUA & CCA & 73 & Leu & Pro \\
$\mathbf{2}$ & $\mathbf{8 4 8}$ & C & T & $\mathbf{4}$ & ACG & AUG & $\mathbf{2 8 3}$ & Thr & Met \\
3 & 1,022 & C & A & 1 & CCU & CAU & 341 & Pro & His \\
4 & 1,177 & G & A & 1 & GAA & AAA & 393 & Glu & Lys \\
5 & 1,178 & A & G & 1 & GAA & GGA & 393 & Glu & Gly \\
\hline
\end{tabular}

In bold the major statistically significant mutation is highlighted. 

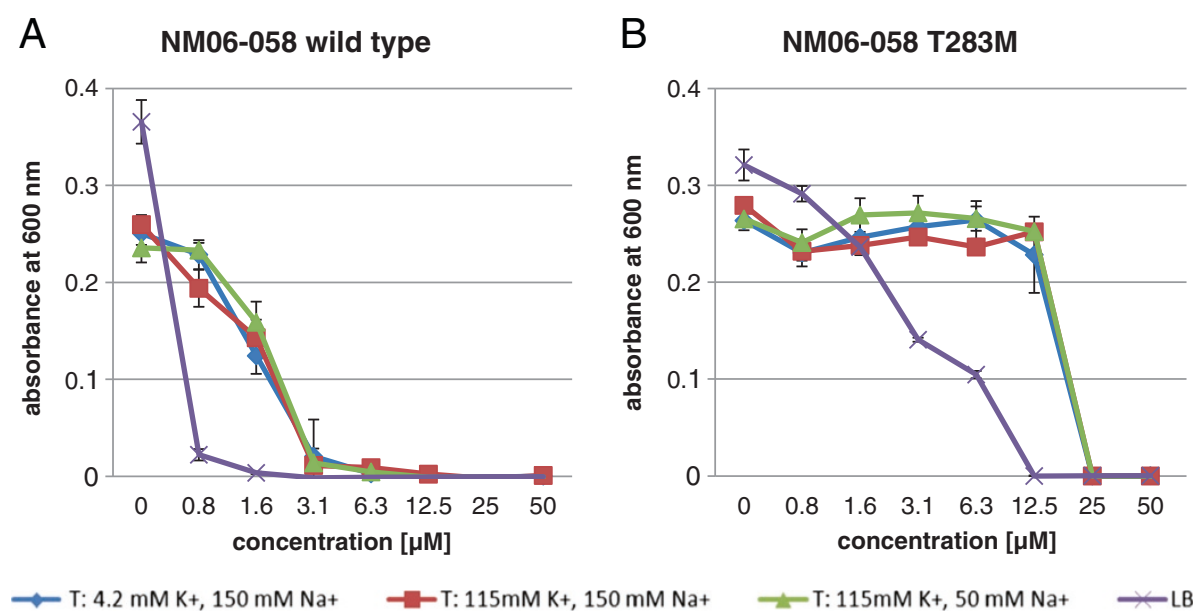

Figure 4 Growth determination. Growth of $V$. cholerae wild type strain NM06-058 (A) and the T283M exchange mutant (B) in the presence of vz0825 in media with different $\mathrm{K}^{+}$and $\mathrm{Na}^{+}$concentrations.

passage indicates a single essential molecular target of vz0825. The aligned sequences of the wild type genome and the mutant genome pool were compared with each other. For the identification of significant mutations the minimal frequency in the mutant genome pool was defined at $30 \%$. A lower frequency would deliver too many non-relevant mutations. In the genome pool of the 15 resistant mutants only the gene with the code number VC_A0531, which corresponds to the homologue $k d p D$ in E.coli, showed a significant mutation under the chosen parameters with frequency of $29.1 \%$. The sequencing of the 15 resistant mutants showed, that 4 of them $(26.7 \%)$ possess this particular modification. The mutated nucleobase is the second base of the corresponding codon and causes an exchange of the amino acid threonin by methionine in the expressed protein. Another four mutants also possess point mutations at other positions of the gene (shown in Figure 5). All of those mutations lead to an exchange of one particular amino acid in the expressed protein, two of them which are located in the N-region (position 1,177 and 1,178 ) lead to the exchange of glutamic acid 393 to lysine or glycin, respectively (Table 7 and Figure 5). Thus, 8 of 15 mutants possess a mutation in the $k d p D$ gene.

A comparison of known protein domains in the database Pfam Protein Families [21] resulted in the localization of the affected amino acid in the dimerization/phosphor acceptor domain. Histidine kinase dimers are formed by parallel association of two domains creating 4-helix bundles; usually these domains contain a conserved histidine residue and are activated via trans-autophosphorylation by the catalytic domain [22]. They subsequently transfer the phosphoryl group to the aspartic acid acceptor residue of a response regulator protein. Based on the comparison of conserved regions in a number of bacterial histidine kinases [20], the localization could be specified more precisely between the $\mathrm{H}$-region and the $\mathrm{N}$-region (Figure 5). The $\mathrm{H}$-region is the most variable sequence of histidine kinases in bacteria and contains the histidine that is phosphorylated in the signal transduction process. The N-region shuttles the gamma-phosphate from ATP to the histidine residue. The mutated amino acid is localized between the conserved $\mathrm{H}$ - and $\mathrm{N}$-region (Figure 5) and thus in a part of the protein that shows high interspecies variation [23], which could explain the specificity of vz0825 against $V$. cholerae.

In the two-component system of signal transduction, the histidine kinase transfers the signal to a response regulator. The $V$. cholerae protein VC_A0531 is the homolog of $\mathrm{KdpD}$ in $E$. coli, the response regulator of which is KdpE [24]. The signal transduction system $\mathrm{KdpABC}$, regulated by $\mathrm{KdpD}$ and $\mathrm{KdpE}$, is part of the osmoregulation machinery in bacteria [15]. Compound vz0825 may exert its mode of action by binding to the histidine kinase $\mathrm{KdpD}$ and thereby inhibiting signal transduction. This would lead to a deficient uptake of potassium. If this mechanism leads to the observed reduction of bacterial viability remains to be elucidated.

Due to a lack of specific information about the potassium regulation in $V$. cholerae, we compared our findings with results that have been obtained with $E$. coli. E. coli possesses in addition to the $\mathrm{KdpABC}$ potassium regulatory system several further $\mathrm{K}^{+}$dependent signal transduction systems. The $\mathrm{K}^{+}$regulatory systems Trk and Kup are active at physiological $\mathrm{K}^{+}$concentrations [15]. The expression of KdpD and consequently of the KdpABC system in $E$. coli is induced at low potassium concentrations $(<60 \mathrm{mM})$ [25]. In E. coli KdpD is not essential at a potassium concentration $>115 \mathrm{mM}$, as mutants with truncated forms of $\mathrm{KdpD}$ are viable under these conditions, but in media with $<15 \mathrm{mM} \mathrm{K}^{+}$those strains do not grow [25]. $\mathrm{V}$. 


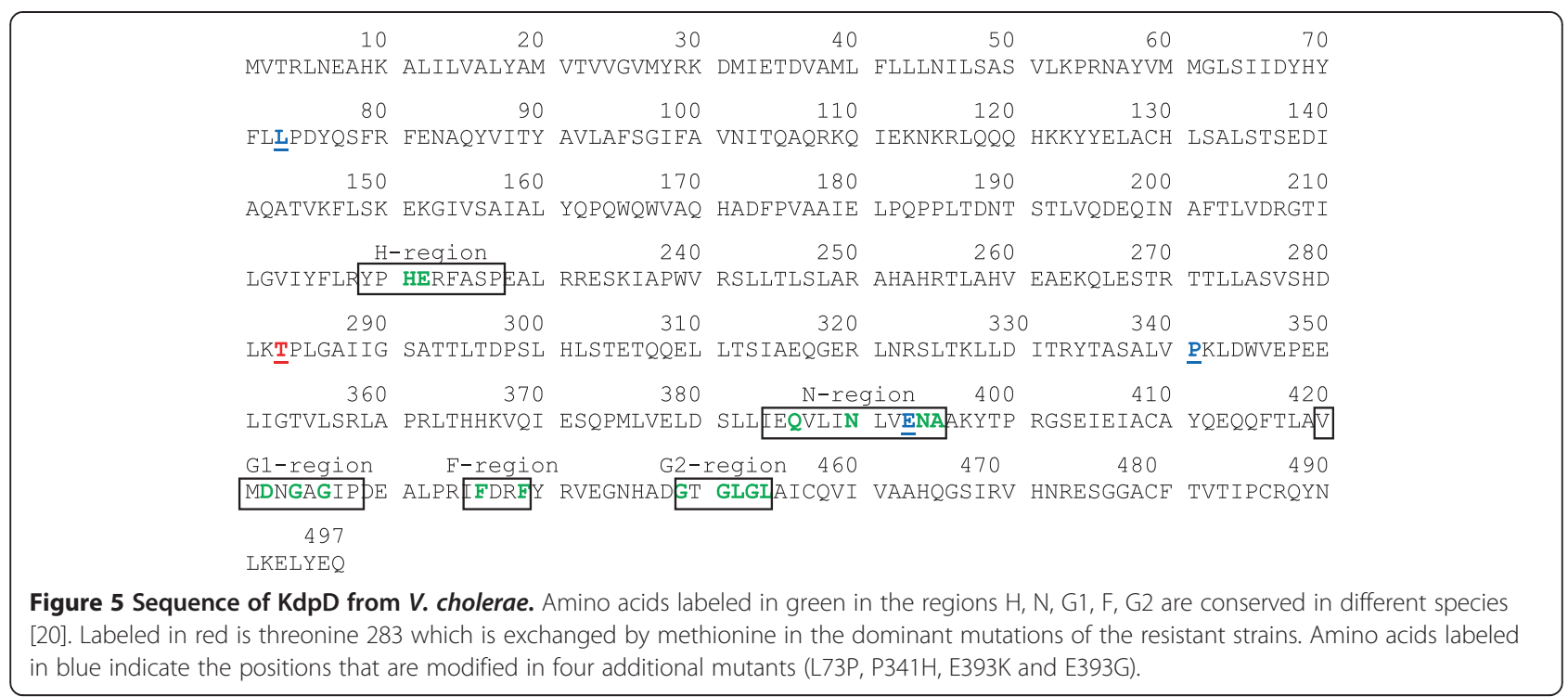

cholerae also possesses these three potassium regulatory systems for the adaptation to changing osmotic conditions [26,27].

The $V$. cholerae mutant strain T283M grows well in media with high and low $\mathrm{K}^{+}$and $\mathrm{Na}^{+}$concentrations in absence of vz0825 as shown in Figure 4 . Even at $4 \mathrm{mM} \mathrm{K}^{+}$ growth is not diminished. This figure also shows the difference between the tolerance of the wild type and the T283M strain against vz0825. Our findings that T283M grows well in $\mathrm{K}^{+}$reduced medium indicates that the inhibition of $\mathrm{KdpD}$ may have profound influence on some other, hitherto undefined, regulatory function of this protein in $V$. cholerae. The influence of vz0825 on KdpD may appear in different ways, e.g. reducing the binding of ATP to the histidine kinase, inhibiting the transfer of gammaphosphate to the histidine residue, or to the asparagine residue of the response regulator. Like other histidine kinases $\mathrm{KdpD}$ also has phosphatase activity [28], which may be disturbed by vz0825. The mutated amino acid on position 283 is located between the $\mathrm{H}$-region and N-region. Mutations that alter this motif, which is termed the $\mathrm{X}$ region, have been shown to alter the conformation of the histidine kinase EnvZ and significantly reduce its phosphatase activity [29]. EnvZ is a membrane receptor kinasephosphatase, which modulates porin expression in $E$. coli in response to medium osmolarity. It shares its basic scheme of signal transduction with many other sensor-kinases [29].

If $\mathrm{KdpD}$ is the major target of compound $\mathrm{vz0} 225$, the deletion construct $\Delta k d p D$ should be insensitive to the substance in media with physiological $\mathrm{K}^{+}$concentration provided that it is still viable. The construction of the required plasmid for the generation of this construct, its transformation into E. coli S17-1 and the conjugation from $E$. coli into $V$. cholerae were successful in this study, but several attempts to induce the homolog recombination within $V$. cholerae NM06-058 failed. None of the analyzed clones showed a loss of the $k d p D$ gene. The apparent growth reducing effect of vz0825 and its targeting of $\mathrm{KdpD}$ in $V$. cholerae suggests a more important role of $\mathrm{KdpD}$ in $V$. cholerae than in E. coli. Further experiments are required in order to corroborate the effect of vz0825 on $\mathrm{KdpD}$, like functional assays with the expressed protein, in which the kinase- and phosphatase activities of the wild type and mutated forms in the presence of vz0825 are compared. It would also be desirable to carry out expression profiling of the transcriptome of vz0825 sensitive and resistant $V$. cholerae strains. This procedure could help to determine how relevant the expression of $k d p D$ in $V$. cholerae is and whether the expression of other genes is reduced or induced in the resistant strains.

\section{Conclusions}

In a high-troughput screening assay with 28,300 compounds the synthetic small molecule vz0825 was identified as the most active antibacterial substance against $V$. cholerae with an MIC of $1.6 \mu \mathrm{M}$ and an MBC of $3.2 \mu \mathrm{M}$. Whole genome sequencing was carried out with resistant mutants and the two-component histidine kinase $\mathrm{KdpD}$ was identified as the prime target of the substance. Further investigations should address the inhibitory mechanism in more detail and corroborate on the possibility of an essential function of $\mathrm{KdpD}$ in $V$. cholerae. Histidine kinase inhibitors are in principal promising antimicrobial drug candidates [30] and compounds like vz0825 may lead to new treatment options.

\section{Methods}

Strains, media and plasmids

The strains used in this study are listed in Table 3. Reporter strain MO10 pG13 was generated from the 
pathogenic wild type strain MO10, serogroup O139, which was electroporated with the plasmid construct pG13 containing a kanamycin resistance gene $\left(\mathrm{Km}^{\mathrm{r}}\right)$ and was selected on a plate containing $30 \mu \mathrm{g} / \mathrm{ml} \mathrm{Km} . V$. cholerae strains were grown in LB medium $(\mathrm{pH} 7.0)$ at $37^{\circ} \mathrm{C}$. LB medium containing $\mathrm{Km}(30 \mu \mathrm{g} / \mathrm{ml})$ was used for HTS and Cip $(100 \mu \mathrm{M})$ was used for positive control. To determine the MIC and MBC values, Mueller-Hinton (MH) broth (pH 7.4) was used as growth medium. Susceptibility to ampicillin (Amp), tetracycline (Tet), Cip, rifampicin, chloramphenicol, erythromycin, sulfamethoxazole, and trimethoprim/sulfamethoxazole (SXT) was determined in 96-well MTP containing MH medium supplemented with varied amounts ( 1 to $1,024 \mu \mathrm{g} / \mathrm{ml}$ ) of each antibiotic separately and varied amounts of SXT (0.13/2.38 to 8/152 $\mu \mathrm{g}$ / $\mathrm{ml})$. Supplemented LB medium with Amp (50 $\mu \mathrm{g} / \mathrm{ml}), \mathrm{Km}$ $(30 \mu \mathrm{g} / \mathrm{ml})$ and Carb $(100 \mu \mathrm{g} / \mathrm{ml})$ was used during the procedures of site-directed mutagenesis and in $\mathrm{T}$ medium $\mathrm{pH}$ 7.4. $\mathrm{T}$ medium was prepared by adding $17 \mathrm{~g}$ tryptone, $3 \mathrm{~g}$ neutralized soy peptone, $10 \mathrm{~g}$ glucose, $50 \mathrm{mM}$ MOPS, $100 \mathrm{mM} \mathrm{NaCl}, 2 \mathrm{mM} \mathrm{KCl}$ and $2 \mathrm{mM} \mathrm{CaCl} 2$ in $1 \mathrm{l}$ of water. For homolog recombination $\mathrm{NaCl}$-free (for increased sucrose sensitivity [31]) LB medium or T medium with $10 \%$ sucrose (for induction of pEX18Ap plasmid excision, carrying the $s a c B$ gene) was used. Cultivation of the mouse fibroblas cell line L292 was carried out in DMEM with 10\% FBS (Lonza).

\section{Substance collections}

Three commercially available substance collections were used in the screening campaigns: i) the LOPAC collection of pharmacologically active compounds with 1,408 entities (Sigma-Aldrich); ii) the Echaz Microcollection with 7,304 compounds (EMC Microcollections $\mathrm{GmbH}$, Tübingen, Germany); and iii) the CDI collection with approximately 17,000 compounds (Chemical Diversity Lab, Inc., San Diego, USA), this commercially available collection has been assembled by members of the ChemBioNet consortium [32]. Three additional libraries that were used are unique at the HZI: iv) the $\mathrm{NCH}$ collection consisting of 154 secondary metabolites from myxobacteria [33]; v) the library Various Sources (VAR) contained at the time of this study 1,936 synthetic organic molecules that were provided by various collaborators; and vi) the Peptide library contained 1,045 short linear or cyclic peptide sequences synthesized at the HZI [6]. All test compounds were utilized as stock solutions in DMSO.

\section{Growth assay}

$50 \mu \mathrm{l}$ or $25 \mu \mathrm{l}$ of LB-Km medium were inoculated in clear flat-bottom 96-well or 384-well MTP, respectively. Test compounds were added from DMSO stocks in amounts that resulted in assay concentrations between 20 and $50 \mu \mathrm{M}$. $50 \mu \mathrm{l}$ or $25 \mu \mathrm{l}$ of bacterial culture in LB-Km medium with an absorbance of 0.2 at $600 \mathrm{~nm}\left(\mathrm{OD}_{600}\right)$ (Ultraspec 2100 Pro photometer, Pharmacia, GE Healthcare, Chalfont St Giles, UK) were added to the 96-well or 384-well MTP, respectively. The seeding of bacteria and addition of the compounds was carried out with the pipetting system Evolution P3 (PerkinElmer, Waltham, USA). Stationary incubation of the plates for $24 \mathrm{~h}$ at $37^{\circ} \mathrm{C}$ under moist conditions was carried out, followed by determination of absorbance at $600 \mathrm{~nm}$ and fluorescence at $485 / 535 \mathrm{~nm}$ (Fusion Universal Microplate Analyzer, PerkinElmer, Waltham, USA). As negative and positive controls DMSO (1\%) and Cip $(100 \mu \mathrm{M})$ were used, respectively. During the initial screening, approximately 28,300 compounds were investigated with single determinations. Compounds that reduced bacterial growth by at least $50 \%$ were retested in a second campaign and the most active substances were reevaluated at different concentrations between 0.1 and $100 \mu \mathrm{M}$.

\section{MIC and $M B C$ values determination}

The determination of MIC and MBC values was carried out with V. cholerae wild type strains and several Gramnegative and Gram-positive bacteria (Table 3) following standardized protocol [34] in broth dilution assays. Starting inocula of $2-8 \times 10^{5}$ colony forming units $/ \mathrm{ml}(\mathrm{CFU} / \mathrm{ml})$ in $\mathrm{MH}$ medium at $37^{\circ} \mathrm{C}$ were used and serial dilutions were carried out in 96-well MTP in duplicate. At 2, 6 and $24 \mathrm{~h}$ of incubation, $10 \mu \mathrm{l}$ of the cultures were plated on LB agar plates. After an incubation of the plates for $24 \mathrm{~h}$ at $37^{\circ} \mathrm{C}, \mathrm{CFU} / \mathrm{ml}$ were determined and used for the determination of $\mathrm{MBC}$, which is defined as minimum concentration of the substance required for $99.9 \%$ reduction of $\mathrm{CFU}$ after an incubation period of $6 \mathrm{~h}$. The $2 \mathrm{~h}$ and $24 \mathrm{~h}$ measurements were used for additional correlation. MIC values were determined after $24 \mathrm{~h}$ of incubation.

\section{Cytotoxicity assay}

The mammalian cell line L929 was utilized to investigate the cytotoxicity of the active compounds in a MTT assay according to a modified protocol of Mosmann [11,12]. Following $24 \mathrm{~h}$ of incubation, acute toxicity was determined based on the extent of cell viability and after incubation for $5 \mathrm{~d}$ mainly the inhibition of cell proliferation and subacute toxicity were measured (absorption at $595 \mathrm{~nm}$ ) (Wallac Victor 1420 Multilabel counter, PerkinElmer, Waltham, USA). $\mathrm{IC}_{50}$ is the concentration that reduces the viability of the cells by $50 \%$.

\section{Generation of resistant mutants against vz0825}

The protocol for the generation of resistant mutants was the same as used in the publication of Bielecki et al. [13]. $V$. cholerae strain NM06-058 was plated at a cell number of $1 \times 10^{9}$ CFU on LB agar plates containing $8 \mu \mathrm{M}$ vz0825 (5times the MIC value). After incubation for $24 \mathrm{~h}$ at $37^{\circ} \mathrm{C}$, 
micro-colonies were visible. 15 colonies were picked and preserved as mutants against vz0825.

\section{Isolation of genomic DNA and sequencing of genome-pool}

Isolation of the genomic DNA was performed according to the protocol of the DNeasy Blood and Tissue Kit (Qiagen). Briefly, the 15 resistant mutants were inoculated individually in $5 \mathrm{ml} \mathrm{LB}$ medium and incubated for $6 \mathrm{~h}$ at $37^{\circ} \mathrm{C}$ with shaking at $180 \mathrm{rpm}$. In parallel, the wild type strain was cultivated under identical conditions. Based on the $\mathrm{OD}_{600}$ measurements of the cultures, the 15 mutants were pooled in equal amounts. After adjusting the cell number at $2 \times 10^{9} \mathrm{CFU}$ the pooled mutants and the wild type strain were collected by centrifugation. The cell pellets were lysed by addition of ATL buffer and proteinase $\mathrm{K}$ for $1 \mathrm{~h}$ at $56^{\circ} \mathrm{C}$. RNA was removed by addition of $4 \mu \mathrm{l}$ RNase A $(100 \mathrm{mg} / \mathrm{ml})$ and incubation for 2 min at RT. $200 \mu \mathrm{l} \mathrm{AL}$ buffer and afterwards $200 \mu \mathrm{l}$ of ethanol were added with mixing. The mixture was transferred to DNeasy Mini spin columns and centrifuged at $\geq 6.000 \times g$ for $1 \mathrm{~min}$. Washing was carried out with $500 \mu \mathrm{l}$ AW1 buffer followed by centrifugation for 1 min. A second washing step was carried out with $500 \mu \mathrm{l}$ AW2 buffer. The tubes were centrifuged for $3 \mathrm{~min}$ at $20,000 \times g$ and the genomic DNA was eluted from the membranes with $200 \mu \mathrm{l} \mathrm{AE}$ buffer.

Whole genome sequencing, alignment and annotation were carried out in the sequencing facility of the HZI (head Dr. Robert Geffers). Libraries of DNA fragments with an average length of $300 \mathrm{bp}$ were prepared according the manufacturer's instructions "Preparing Samples for Sequencing Genomic DNA" (Illumina). Sequencing was carried out with the Illumina Cluster Station and the Genome Analyzer IIx. The resulting data was transformed into FastQ-format. Sequencing of the DNA library resulted in a total base count of 855,825,664 and 2,546,713,435 for wild type and resistant mutants genome pool, respectively. This corresponds to a calculated average coverage of 214 for the wild type and for each resistant mutant to a coverage of 42 . The published complete genome has a total base number of 4,033,460 (Table 6, [14]).

The sequencing procedure resulted in 11,260,862 and $35,196,596$ reads for wild type and resistant mutants genome pools, respectively, which were mapped to the reference genome of the annotated $V$. cholerae strain N16961 [14] by the application of the Read Mapper Tool and the Probabilistic Variant Caller as part of CLC Genomics Workbench V. 4.7.2 software. The Read Mapper Tool maps reads and calculates average coverage at single nucleotide resolution. The Probabilistic Variant Caller identifies variants by using a probabilistic model built from read mapping data. Based on a combination of a Bayesian model and a Maximum Likelihood approach the algorithm calculates prior and error probabilities for the Bayesian model. By using the Probabilistic Variant Caller software and defining various parameters, such as sequence frequency, size of mutated areas and mutation abundance, lists of SNPs and DIPs were created. A frequency of more than 30 reads was required for all fragments. The maximum number of allelvariations was restricted to two, and the threshold of the frequency of the allel-variations was set at a minimum of $30 \%$. These lists were compared for the wild type strain and the pooled resistant mutants, and SNPs that are unique for the mutants were identified.

\section{Colony PCR and sequencing}

The 15 resistant mutants were analyzed individually to determine whether they carry the point mutation on position 848 of the $k d p D$ gene. Individual colonies were heated in $36.5 \mu \mathrm{l}$ of water for $5 \mathrm{~min}$ at $95^{\circ} \mathrm{C} .1 \mu \mathrm{l}$ of dNTPs (stock solution $10 \mathrm{mM}$ ), $2.5 \mu \mathrm{l}$ of primers VC_A0531_forw2 and VC_A0531_rev2 (stock solution $100 \mathrm{pmol} / \mu \mathrm{l}), 5 \mu \mathrm{l} 10 \times$ PCR buffer and $2.5 \mu \mathrm{l}$ RED Taq polymerase $(1 \mathrm{U} / \mu \mathrm{l})$ were added. After the PCR procedure, the products had the expected size of $915 \mathrm{bp}$. They were purified and sequenced in the sequencing facility of the HZI using the above primers.

\section{Construction of the point-mutant KdpD T283M in strain NM06-058}

The gene VC_A0531 has a size of 1,494 base pairs (coding for 497 amino acids plus stop codon). The base cytosine, which was changed to tyrosine in the predominant resistant mutants, is located on position 848. Sitedirected mutagenesis was used for the incorporation of this modification into the wild type strain NM06-058. Two overlapping amplicons with a size of 525 and $616 \mathrm{bp}$ were generated from the gene of the wild type strain NM06-058. Fragment one was amplified using the primer pair (i) Mut_forw_1/Mut_rev_1, and the second fragment was amplified with primer pair (ii) Mut_forw_2/Mut_rev_2. The primers Mut_rev_1 and Mut_forw_2 carried the point-mutation (Table 3, bold nucleobases). Primers Mut_forw_1 and Mut_rev_2 contained specific recognition nucleotide sequences for the

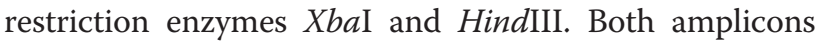
were mixed at equimolar ratio and a re-PCR was performed with the primers Mut_forw_1 and Mut_rev_2 to generate an amplicon with a size of $1,114 \mathrm{bp}$. This amplicon and the plasmid pEX18Ap were restricted with $X b a \mathrm{I}$ and HindIII. Insert and plasmid were ligated and transformed into chemically competent $E$. coli strain S17-1. Amp $(100 \mu \mathrm{g} / \mathrm{ml})$ was incorporated into the agar of the plate for selection of pEX18Ap containing transformants. PCR based analysis of the transformants followed by nucleotide sequencing analysis confirmed the proper insert into the vector, which was subsequently used for the conjugation assay. 
Conjugation was carried out on LB agar plates overnight with a bacterial proportion of 4:1 of E. coli containing conjugative plasmid (donor) and $V$. cholerae as recipient strain. Bacterial cultures (mixed E. coli and V. cholerae) were plated on LB agar plate containing Carb $(100 \mu \mathrm{g} / \mathrm{ml})$ and $\mathrm{Km}(30 \mu \mathrm{g} / \mathrm{ml})$ for selection of $V$. cholerae transconjugants carrying the plasmid. The removal of vector backbone from $V$. cholerae genome was achieved by favoring the homologous recombination and use of lethal $s a c B$ gene while passaging the transconjugants in sodium chloride free LB medium supplemented with $10 \%$ sucrose.

\section{Attempts for construction of a $k d p D$ knockout mutant using $V$. cholerae strain NM06-058}

The gene VC_A0531 encodes for the histidine kinase $\mathrm{KdpD}$ in $V$. cholerae and is flanked by the genes VC_A0530 encoding pyruvate-flavoredoxin oxidoreductase and VC_A0532 encoding response regulator $\mathrm{KdpE}$ homologue of E. coli. To generate a VC_A0531 deletion mutant, two fragments were amplified from the small chromosome of the wild type strain NM06-058 using two primer pairs (i) $k d p D \_$del_forw_1 / $k d p D \_d e l \_r e v \_1$ and (ii) $k d p D \_$del_forw_2 / $k d p D \_$del_rev_2. Using the first primer pair an approximately $600 \mathrm{pb}$ fragment of gene VC_A0530 was amplified with a 24 bp homolog overhang to the start region of the VC_A0532 at the Cterminus. The second primer pair was used to amplify an approximately $400 \mathrm{bp}$ fragment of the gene VC_A0532 with a $16 \mathrm{bp}$ overhang homolog to the end region of the VC_A0530 at the N-terminus. Both amplicons were mixed together at equimolar ratio and a re-PCR was carried out with a combination of primers $k d p D$ _del_forw_1 and $k d p D \_d e l \_r e v \_2$ to generate an amplicon with a size of approximately $1,000 \mathrm{bp}$. The restriction of vector pEX18Ap and the insert was carried out with XbaI and PstI. After ligation and transformation into E. coli $\mathrm{S} 17-1$, a conjugation into the wild type $V$. cholerae strain NM06-058 was mediated according to the protocol described above. The cloning strategy was successful until transconjugation according selection on Carb / Km agar plates and sequencing, but homolog recombination attempts with $V$. cholerae strain NM06-058 did not yield viable strains with deleted $k d p D$ gene.

\footnotetext{
Abbreviations

AA: Amino acid(s); Amp: Ampicillin; Carb: Carbenicillin; Cip: Ciprofloxacin; CFU: Colony-forming unit; CT: Cholera toxin; DIP: Deletion and insertion polymorphism; DMSO: Dimethyl sulfoxide; ER: Endoplasmic reticulum; GFP: Green Fluorescent Protein; HTS: High-throughput screening; Km: Kanamycin; MBC: Minimal bactericidal concentration; MIC: Minimal inhibitory concentration; MTP: Microtiter plate; RKI: Robert Koch Institute; SNP: Single nucleotide polymorphism; SXT: Trimethoprim/sulfamethoxazole; Tet: Tetracycline.
}

\section{Competing interests}

The authors declare that they have no competing interests.

\section{Authors' contributions}

GS performed experiments, including assay development, screening, hit evaluation and the first target analysis using genome sequencing of resistant mutants. MJ is member of the sequencing facility at the $\mathrm{HZI}$ and carried out and interpreted the genome sequencing. SR developed the reporter strain MO10 pG13 which was used for the screening. Compounds showing activity against $V$. cholerae were conceived and synthesized by DT and VAZ. RKN and WT conceived the study, participated in its design and coordination and helped to draft or revise the manuscript. All authors read and approved the final manuscript.

\section{Acknowledgments}

Authors thankfully acknowledge helpful technical assistance from Subhasis Barik. We thank the Indian Council of Medical Research (ICMR), Govt. of India and for funding support through the Indo-German Science Centre (Sanction No. TDR/491/2008-ECD-II). SR is recipient of a Junior Research Fellowship from the Council of Scientific and Industrial Research (CSIR), Govt. of India (Sanction No. 09/482(0054)/2010-EMR-I).

\section{Author details}

${ }^{1}$ Department of Chemical Biology, Helmholtz Centre for Infection Research (HZl), Inhoffenstraße 7, D-38124 Braunschweig, Germany. ${ }^{2}$ National Institute of Cholera and Enteric Diseases (NICED), P-33, CIT Road, Scheme XM Beliaghata, Kolkata 700 010, India. ${ }^{3}$ Technical University of Clausthal, Leibnizstraße 6, D-38678 Clausthal-Zellerfeld, Germany.

Received: 2 August 2013 Accepted: 19 February 2014 Published: 26 February 2014

\section{References}

1. WHO: Cholera. In Fact sheet No 107; 2011. http://www.who.int/mediacentre/ factsheets/fs107/en/.

2. Kitaoka M, Miyata ST, Unterweger D, Pukatzki S: Antibiotic resistance mechanisms of Vibrio cholerae. J Med Microbiol 2011, 60(4):397-407.

3. Zhang JH, Chung TDY, Oldenburg KR: A simple statistical parameter for use in evaluation and validation of high throughput screening assays. J Biomol Screen 1999, 4(2):67-73.

4. Bollag DM, McQueney PA, Zhu J, Hensens O, Koupal L, Liesch J, Goetz M, Lazarides E, Woods CM: Epothilones, a new class of microtubulestabilizing agents with a taxol- like mechanism of action. Cancer Res 1995, 55(11):2325-2333.

5. Höfle G, Bedorf N, Steinmetz H, Schomburg D, Gerth K, Reichenbach H: Epothilone A and B-novel 16-membered macrolides with cytotoxic activity: Isolation, crystal structure, and conformation in solution. Angewandte Chemie (International Edition in English) 1996, 35(13-14):1567-1569.

6. Tegge W, Bautsch W, Frank R: Synthesis of cyclic peptides and peptide libraries on a new disulfide linker. J Pept Sci 2007, 13(10):693-699.

7. Weissman KJ, Müller R: A brief tour of myxobacterial secondary metabolism. Bioorganic Med Chem 2009, 17(6):2121-2136.

8. Feng Y, Chen CJ, Su LH, Hu S, Yu J, Chiu CH: Evolution and pathogenesis of Staphylococcus aureus: Lessons learned from genotyping and comparative genomics. FEMS Microbiol Rev 2008, 32(1):23-37.

9. Barker LP, Porcella SF, Wyatt RG, Small PLC: The Mycobacterium marinum G13 promoter is a strong sigma 70-like promoter that is expressed in Escherichia coli and mycobacteria species. FEMS Microbio/ Lett 1999, 175(1):79-85.

10. Huang Y, Wang J, Li G, Zheng Z, Su W: Antitumor and antifungal activities in endophytic fungi isolated from pharmaceutical plants Taxus mairei, Cephalataxus fortunei and Torreya grandis. FEMS Immunol Med Microboil 2001, 31(2):163-167.

11. Mosmann T: Rapid colorimetric assay for cellular growth and survival: Application to proliferation and cytotoxicity assays. J Immunol Methods 1983, 65(1-2):55-63.

12. Sasse F, Steinmetz H, Schupp T, Petersen F, Memmert K, Hofmann $H$, Heusser C, Brinkmann V, Von Matt P, Höfle G, Reichenbach H: Argyrins, immunosuppressive cyclic peptides from myxobacteria - I. Production, isolation, physico-chemical and biological properties. J Antibiot (Tokyo) 2002, 55(6):543-551.

13. Bielecki $P$, Lukat $P$, Hüsecken $K$, Dötsch $A$, Steinmetz H, Hartmann RW, Müller R, Häussler S: Mutation in elongation factor $G$ confers resistance to the antibiotic argyrin in the opportunistic pathogen pseudomonas aeruginosa. Chem Bio Chem 2012, 13(16):2339-2345. 
14. Heidelberg JF, Elsen JA, Nelson WC, Clayton RA, Gwinn ML, Dodson R, Haft DH, Hickey EK, Peterson JD, Umayam L, Gill SR, Nelson KE, Read TD, Tettelin H, Richardson D, Ermolaeva MD, Vamathevan J, Bass S, Halving Q, Dragol I, Sellers P, McDonald L, Utterback T, Fleishmann RD, Nierman WC, White O, Saizberg SL, Smith HO, Colwell RR, Mekalanos JJ: DNA sequence of both chromosomes of the cholera pathogen Vibrio cholerae. Nature 2000, 406(6795):477-483.

15. Csonka LN, Hanson AD: Prokaryotic osmoregulation: genetics and physiology. Annu Rev Microbiol 1991, 45:569-606.

16. Ishii I, Katagir M, Sakazume K, Misato T: Antibacterial effect of photosensitising dyes on Xanthomonas oryzae, leaf blight bacteria on rice plants. I. The relationship between the chemical structure of dyes and their antibacterial activity [in Japanese]. Nippon Vogei Kagaku Kaishi 1966, 40:437-442.

17. Gwynn MN, Portnoy A, Rittenhouse SF, Payne DJ: Challenges of antibacterial discovery revisited. Ann N Y Acad Sci 2010, 1213:5-19.

18. Drews J: Drug discovery: a historical perspective. Science 2000 287(5460):1960-1964.

19. Raychoudhuri A, Patra T, Ghosh K, Ramamurthy T, Nandy RK, Takeda Y, Nair GB, Mukhopadhyay AK: Classical ctxB in Vibrio cholerae 01, Kolkata, India. Emerg Infect Dis 2009, 15(1):131-132.

20. Parkinson JS, Kofoid EC: Communication modules in bacterial signaling proteins. Ann Rev Gen 1992, 26:71-112.

21. Punta $M$, Coggill $P C$, Eberhardt RY, Mistry J, Tate J, Boursnell C, Pang N Forslund K, Ceric G, Clements J, Heger A, Holm L, Sonnhammer ELL, Eddy SR, Bateman A, Finn RD: The Pfam protein families database. Nucleic Acids Res 2012, 40(D1):D290-D301.

22. Dutta R, Qin L, Inouye M: Histidine kinases: Diversity of domain organization. Mol Microbiol 1999, 34(4):633-640.

23. Stock AM, Robinson VL, Goudreau PN: Two-component signal transduction. Annu Rev Biochem 2000, 69:183-215.

24. Heermann R, Weber A, Mayer B, Ott M, Hauser E, Gabriel G, Pirch T, Jung K: The universal stress protein UspC scaffolds the $\mathrm{KdpD} / \mathrm{KdpE}$ signaling cascade of Escherichia coli under salt stress. J Mol Biol 2009, 386(1):134-148.

25. Puppe W, Zimmann P, Jung K, Lucassen M, Altendorf K: Characterization of truncated forms of the KdpD protein, the sensor kinase of the $\mathrm{K} \pm-$ translocating Kdp system of Escherichia coli. J Biol Chem 1996 271(40):25027-25034.

26. The Kup system in Vibrio cholerae. [http://www.ncbi.nlm.nih.gov/gene/? term=kup+vibrio+cholerae]

27. The Trk system of Vibrio cholerae. [http://www.ncbi.nlm.nih.gov/gene/? term=trk+vibrio+cholerae]

28. Brandon L, Dorus S, Epstein W, Altendorf K, Jung K: Modulation of KdpD phosphatase implicated in the physiological expression of the Kdp ATPase of Escherichia coli. Mol Microbiol 2000, 38(5):1086-1092.

29. Hsing W, Russo FD, Bernd KK, Silhavy TJ: Mutations that alter the kinase and phosphatase activities of the two-component sensor EnvZ. J Bacteriol 1998, 180(17):4538-4546.

30. Matsushita M, Janda KD: Histidine kinases as targets for new antimicrobial agents. Bioorganic Med Chem 2002, 10(4):855-867.

31. Blomfield IC, Vaughn V, Rest RF, Eistenstein BI: Allelic exchange in Escherichia coli using the Bacillus subtilis sacB gene and a temperaturesensitive pSC101 replicon. Mol Microbiol 1991, 5(6):1447-1457.

32. Lisurek M, Rupp B, Wichard J, Neuenschwander M, Von Kries JP, Frank R, Rademann J, Kühne R: Design of chemical libraries with potentially bioactive molecules applying a maximum common substructure concept. Mol Divers 2010, 14(2):401-408.

33. Gerth K, Pradella S, Perlova O, Beyer S, Müller R: Myxobacteria: proficient producers of novel natural products with various biological activities Past and future biotechnological aspects with the focus on the genus Sorangium. J Biotechnol 2003, 106(2-3):233-253.

34. DIN 58940-7: Medical microbiology - susceptibility testing of microbial pathogens to antimicrobial agents - determination of the minimum bactericidal concentration $(\mathrm{MBC})$ with the method of microbouillondilution; text in German and English. 2009. http://webstore.ansi.org/RecordDetail. aspx?sku=DIN+58940-7\%3A2009.

doi:10.1186/1471-2180-14-49

Cite this article as: Sergeev et al:: High-throughput screening and whole genome sequencing identifies an antimicrobially active inhibitor of Vibrio cholerae. BMC Microbiology 2014 14:49.

\section{Submit your next manuscript to BioMed Central and take full advantage of:}

- Convenient online submission

- Thorough peer review

- No space constraints or color figure charges

- Immediate publication on acceptance

- Inclusion in PubMed, CAS, Scopus and Google Scholar

- Research which is freely available for redistribution 